(Preprint) AAS 17-286

\title{
TRAJECTORY DESIGN FOR A CISLUNAR CUBESAT LEVERAGING DYNAMICAL SYSTEMS TECHNIQUES: THE LUNAR ICECUBE MISSION
}

\author{
Natasha Bosanac; Andrew Cox $₫$ Kathleen C. Howell $;$ and David C. Folta ${ }^{\S}$
}

\begin{abstract}
Lunar IceCube is a $6 \mathrm{U}$ CubeSat that is designed to detect and observe lunar volatiles from a highly inclined orbit. This spacecraft, equipped with a low-thrust engine, will be deployed from the upcoming Exploration Mission-1 vehicle in late 2018. However, significant uncertainty in the deployment conditions for secondary payloads impacts both the availability and geometry of transfers that deliver the spacecraft to the lunar vicinity. A framework that leverages dynamical systems techniques is applied to a recently updated set of deployment conditions and spacecraft parameter values for the Lunar IceCube mission, demonstrating the capability for rapid trajectory design.
\end{abstract}

\section{INTRODUCTION}

With the emergence and increased development of miniaturized satellite technologies, CubeSats offer an alternative platform for unmanned exploration of cislunar space and, eventually, the solar system. ${ }^{1,2,3}$ In fact, their reduced cost and development time in contrast to larger, traditional spacecraft supports a wide array of organizations conducting scientific observation, technology demonstration and space exploration missions. Continued advancement in these small satellite technologies demands the innovative design of trajectories for spacecraft that require fewer resources, achieve more complex mission goals and visit even farther destinations. Furthermore, small spacecraft typically ride as secondary payloads onboard a launch vehicle, producing significant uncertainty and variability in the launch date and deployment status. However, such uncertainty in the deployment state of a small spacecraft can severely constrain and complicate the trajectory design process during both the development and operational phases of the mission. Accordingly, the construction of a framework that leverages dynamical systems techniques and enables efficient, rapid and well-informed trajectory design is warranted. ${ }^{4,5}$

To demonstrate the value of a trajectory design framework, consider the Lunar IceCube mission led by Morehead State University and supported by NASA Goddard Space Flight Center (GSFC), Busek and Catholic University of America. The mission concept is based on the use of a $6 \mathrm{U}$ CubeSat which is designed to detect and observe water near the Moon's equatorial regions. ${ }^{2}$ To achieve these scientific goals, an inclined, low altitude lunar elliptical orbit is required during the observational phase of the mission. Currently, Lunar IceCube is scheduled for launch in late 2018 as a secondary payload onboard the upcoming Exploration Mission-1 (EM-1) vehicle. Following deployment close to the Earth, the Lunar IceCube spacecraft is deployed to a high energy trajectory that would naturally depart the Earth-Moon system. However, the spacecraft features a low-thrust propulsion system that is currently estimated to deliver up to $1.15 \mathrm{mN}$ of thrust

* Post-Doctoral Research Assistant, School of Aeronautics and Astronautics, Purdue University, 701 W. Stadium Ave., West Lafayette, Indiana 47907, USA; currently Assistant Professor, Department of Aerospace Engineering Sciences, University of Colorado Boulder, 429 UCB, Boulder, Colorado 80309, USA.

${ }^{\dagger}$ Graduate Student, School of Aeronautics and Astronautics, Purdue University, 701 W. Stadium Ave., West Lafayette, Indiana 47907, USA.

${ }^{\ddagger}$ Hsu Lo Distinguished Professor of Aeronautics and Astronautics, School of Aeronautics and Astronautics, Purdue University, 701 W. Stadium Ave., West Lafayette, Indiana 47907, USA.

$\S$ Senior Fellow, NASA Goddard Space Flight Center, Greenbelt, Maryland 20771, USA. 
with an $I_{s p}$ of 2500s. Finite duration maneuvers can be leveraged, in combination with the natural dynamical structures associated with the gravity of the Sun, Earth and Moon, to design a complex path that delivers the Lunar IceCube spacecraft to its final lunar science orbit. ${ }^{4}$ However, the constrained departure conditions, i.e., the launch date and deployment state, as well as the reduced propulsive capability, may limit both the geometry and persistence of transfers within the phase space. Furthermore, as these departure conditions evolve during both the development and operational phases of the primary mission, a complete trajectory redesign may be required, necessitating the development of a framework for rapidly constructing an initial guess for a transfer that may satisfy the mission and hardware requirements.

To construct a trajectory design framework for a CubeSat with limited propulsive capability and constrained departure conditions, techniques from dynamical systems theory are leveraged. In fact, natural dynamical structures, along with Poincaré mapping strategies, are employed to design a complex path that delivers the spacecraft from a highly energetic deployment state to the final lunar science orbit. ${ }^{4}$ With application to the Lunar IceCube mission, analysis of existing point solutions reveals that the transfer trajectory can be split into three components: an Earth outbound segment, a phasing and energy adjustment segment that exploits solar gravity and, finally, lunar approach and capture. ${ }^{4,10}$ The first and final segments rely heavily on the low-thrust propulsion system to modify the spacecraft energy. However, given the limitations on the propulsive capability of the Lunar IceCube spacecraft, predominantly natural arcs are sought to link these two bounding segments. In addition, the phasing and energy adjustment segment exhibits the largest variability in terms of the available geometries and trajectory characteristics. To effectively design this transfer segment, a circular restricted three-body model of the Sun-Earth system is employed to supply insight into the geometry of natural dynamical structures that can be incorporated into the design of an end-to-end path. Furthermore, approximate bounds on the motion are established and transfer geometries are understood via analysis of the manifolds of libration point orbits. ${ }^{10}$ These natural motions are visualized and linked to the deployment and final lunar science orbit bounding conditions via Poincaré mapping strategies, thereby enabling rapid and efficient construction of a discontinuous initial guess. Depending on the deployment conditions, that frequently evolve throughout further development of the mission concept, the exact definition of the constructed maps may require updates; however, a general framework that leverages mapping techniques remains useful. A differential corrections process is then formulated in a low-thrust-enabled ephemeris model to identify an end-to-end trajectory that generally retains the characteristics of the initial guess. ${ }^{10}$ The resulting continuous solution can then be input to an operational-level model for further corrections or even an optimization algorithm. The constructed trajectory design framework, employed to link the highly energetic deployment state of the Lunar IceCube spacecraft to the final lunar science orbit, supports the identification of feasible transfer regions and rapid redesign. Implementation of this strategy eliminates the challenges associated with a grid search in a nonlinear and chaotic system. Moreover, this framework may be applicable to future CubeSat missions that must meet alternative mission goals, such as re-encountering the Moon with a specific energy and/or flight duration, attaining specific Sun-Earth or Earth-Moon orbits, or achieving a heliocentric trajectory that encounters an asteroid, as well as mission extensions.

\section{LUNAR ICECUBE MISSION OVERVIEW}

The Lunar IceCube mission leverages a $6 \mathrm{U}$ CubeSat developed by Morehead State University and supported by NASA Goddard Space Flight Center (GSFC), Busek and Catholic University of America. Specifically, the primary objective for this mission is to observe the equatorial regions of the Moon, furthering knowledge of the location and transportation physics associated with water in various forms. ${ }^{2}$ The scientific instruments onboard the Lunar IceCube spacecraft require that these measurements of lunar water and volatiles be collected from a low lunar science orbit. Currently, the nominal science orbit is highly elliptical with a perilune located over the equator at an altitude between 100 and $105 \mathrm{~km}$. Furthermore, the orbit is also constrained to possess an inclination of approximately 90 degrees. The Lunar IceCube spacecraft, depicted in Figure 1 and currently estimated to possess an initial mass of $14 \mathrm{~kg}$, will ride onboard the Exploration Mission-1 (EM-1) vehicle as a secondary payload. The spacecraft is also equipped with an iodine-fuelled low-thrust engine, a Busek Ion Thruster 3-cm (BIT-3) system, which is currently designed to deliver up to $1.15 \mathrm{mN}$ of thrust with an $I_{s p}$ of $2500 \mathrm{~s}$ and a propellant mass of approximately $1.5 \mathrm{~kg}$. ${ }^{6}$ Previously-developed 


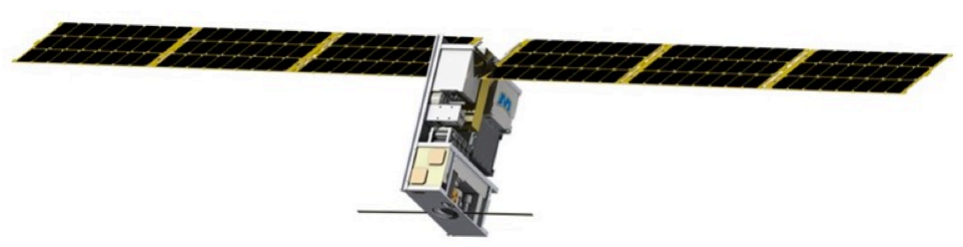

Figure 1: Lunar IceCube preliminary spacecraft design.

trajectory point designs that leverage this low-thrust engine have revealed distinctly different trajectories in a large design space that is difficult to examine through the construction of single point solutions in an ephemeris environment, particularly as the spacecraft hardware and mission parameters evolve. ${ }^{4}$ Thus, a framework employing dynamical systems techniques is used to construct similar solutions based on insight into the natural structures within the gravitational environment of the Earth, Sun and Moon.

\section{DYNAMICAL MODELS}

\section{Circular Restricted Three-Body Problem}

Motion within the Sun-Earth system is rapidly and reasonably approximated using the autonomous dynamics of the Circular Restricted Three-Body Problem (CR3BP). In this dynamical environment, the motion of a massless spacecraft is modeled under the influence of the point-mass gravitational attractions of two primaries: the Sun and the Earth. To enable clear visualization and identification of particular solutions, the motion of the spacecraft is described using a rotating coordinate system. This frame, $(x, y, z)$, rotates with the primaries as they encircle their mutual barycenter. In addition, position and velocity states locating the spacecraft are nondimensionalized. By convention, both the normalized distance between the Sun and the Earth and the mean motion of the primaries are unity. Mass quantities are nondimensionalized such that the masses of the Sun and the Earth are equal to $1-\mu$ and $\mu$, respectively. Using these nondimensionalized quantities, the equations of motion of the spacecraft, located at $(x, y, z)$ in the rotating frame, are compactly written as:

$$
\ddot{x}-2 \dot{y}=\frac{\partial U^{*}}{\partial x}, \quad \ddot{y}+2 \dot{x}=\frac{\partial U^{*}}{\partial y}, \quad \ddot{z}=\frac{\partial U^{*}}{\partial z}
$$

where $U$ is the pseudo-potential function, $U=\frac{1}{2}\left(x^{2}+y^{2}\right)+\frac{1-\mu}{r}+\frac{\mu}{d}$, and $d=\sqrt{(x+\mu)^{2}+y^{2}+z^{2}}, r=$ $\sqrt{(x-1+\mu)^{2}+y^{2}+z^{2}}$. When formulated in the rotating frame, this autonomous dynamical environment admits a constant energy-like integral labeled the Jacobi constant, $C_{J}=2 U-\dot{x}^{2}-\dot{y}^{2}-\dot{z}^{2}$. At a fixed value of this integral, an infinite number of trajectories exist within the Sun-Earth system. Any state along a time-varying solution is defined as prograde if the corresponding angular momentum vector at that instant possesses a $+z$ component, i.e., the spacecraft is traveling in a counterclockwise direction as viewed in the rotating frame of the CR3BP. Correspondingly, a state along a path that possesses a $-z$ component of the angular momentum vector is labeled retrograde. Regardless of the direction of motion, these particular solutions exhibit one of four types of behavior - equilibrium points, periodic orbits, quasi-periodic orbits or chaotic motion - that may be leveraged to design a complex trajectory within the nonlinear gravitational environment of the Earth, Moon and Sun.

\section{Low-Thrust-Enabled Motion in an Ephemeris Model}

To support recovery of a representative end-to-end trajectory, equations of motion are formulated for a spacecraft traveling under the point mass gravitational influences of the Earth, Moon and Sun, as well as an additional acceleration due to a low-thrust propulsion system, leveraging a spacecraft-centered velocitynormal-conormal coordinate frame to describe the direction of the thrust vector. ${ }^{10}$ First, consider $N_{e}$ bodies with dimensional mass $\tilde{M}_{i}$, each assumed to be spherically symmetric and, therefore, modeled as a point mass. These bodies are located in an inertial reference frame, $\hat{X} \hat{Y} \hat{Z}$, with a fixed origin at point $O$. Then, the dimensional position vector locating the body of interest $P_{3}$, at the inertial coordinates $\overline{\tilde{R}}_{3}=(\tilde{X}, \tilde{Y}, \tilde{Z})$, 
with respect to the body $P_{j}$, at the coordinates $\overline{\tilde{R}}_{j}=\left(\tilde{X}_{j}, \tilde{Y}_{j}, \tilde{Z}_{j}\right)$, is found as $\overline{\tilde{R}}_{j 3}=\left(\tilde{X}-\tilde{X}_{j}\right) \hat{X}+(\tilde{Y}-$ $\left.\tilde{Y}_{j}\right) \hat{Y}+\left(\tilde{Z}-\tilde{Z}_{j}\right) \hat{Z}$. To formulate the differential equations governing natural motion within the point mass ephemeris model, the acceleration of the body of interest, $P_{3}$, is expressed relative to a celestial body $P_{q}$ in $P_{q}$-centered J2000 inertial coordinates. ${ }^{7}$ When $P_{3}$ represents a spacecraft, $\tilde{M}_{3} \ll \tilde{M}_{q}, \tilde{M}_{j}$. Thus, the spacecraft is assumed to only negligibly impact the paths of the celestial bodies. Accordingly, additional equations of motion are not required for the $N_{e}-1$ celestial bodies if ephemerides are available.

Prior to augmenting the point mass ephemeris model equations of motion with the additional acceleration contributed by a propulsion system, the characteristics of a low-thrust engine are summarized. Specifically, a constant specific impulse low-thrust engine can be described by its thrust magnitude, $T_{l t}$, specific impulse, $I_{s p}$, and the thrust direction, $\hat{u}$. For this investigation, the iodine-fuelled BIT-3 system onboard the Lunar IceCube spacecraft is assumed to operate with the following parameters: $T_{l t}=0.9 \mathrm{mN}$ and $I_{s p}=2500 \mathrm{~s}$. ${ }^{6}$ It is also assumed that the mass of the spacecraft, $\tilde{m}(t)$, is initially equal to $14 \mathrm{~kg}$. When the low-thrust engine is activated, the spacecraft mass decrements by the following mass flow rate, $\frac{d \tilde{m}(\tilde{t})}{d \tilde{t}}=-\frac{T_{l t}^{2}}{2 P}$, where the constant value for the available engine power is calculated as $P=\frac{T_{l t} I_{s p} g_{0}}{2}$ for a gravitational acceleration, $g_{0}=9.81 \mathrm{~m} / \mathrm{s}^{2}{ }^{8}$ This constant mass flow rate impacts the maximum time for operation of the low-thrust engine which is constrained by the initial propellant mass, assumed to be equal to $1.5 \mathrm{~kg}$. ${ }^{4}$

In this analysis, the direction of the thrust vector is described using a velocity-normal-conormal (VNC) coordinate system, which is often employed for spacecraft applications. ${ }^{9}$ Specifically, the velocity direction, denoted by the $\hat{V}$ unit vector is parallel to the inertial velocity vector of the spacecraft relative to a reference body. Then, the conormal direction, $\hat{C}$, is defined normal to the path of the spacecraft, while lying within the orbital plane. Finally, the normal unit vector, $\hat{N}$, is perpendicular to the orbital plane and completes the righthanded coordinate system. The engine thrust direction, $\hat{u}$, can then be projected into the VNC coordinate system and expressed using these three unit vector directions such that $\hat{u}=u_{V} \hat{V}+u_{N} \hat{N}+u_{C} \hat{C}$. ${ }^{10}$ The thrust vector, $\hat{u}_{V N C}$, expressed in the VNC frame can subsequently be converted into the $P_{q}$-centered J2000 inertial coordinates to produce, $\hat{u}_{X Y Z}$. This transformation is leveraged to incorporate a thrust vector that possesses a fixed direction in the VNC frame into the equations of motion for a point mass ephemeris model.

The additional acceleration for a low-thrust engine is incorporated into the differential equations governing the motion of a spacecraft in a point mass ephemeris model including the Sun, Earth and Moon. First, to mitigate ill-conditioning between the position, velocity and time quantities in the equations of motion, nondimensionalization is employed. Specifically, the characteristic quantities $m^{*}, t^{*}$, and $l^{*}$, computed in the CR3BP for a primary system consisting of the bodies $P_{p}$ and $P_{q}$, may be employed. These nondimensional quantities are denoted without a tilde. Then, the acceleration due to the low-thrust engine is expressed in vector form as $T_{l t}^{*} / \tilde{m}(t) \hat{u}$ where the dimensional mass of the spacecraft, $\tilde{m}$, is employed, and the symbol $T_{l t}^{*}$ represents the thrust magnitude nondimensionalized only in the length and time units for dimensional consistency such that $T_{l t}^{*}=T_{l t} \times\left(t^{*}\right)^{2} / l^{*}$. Then, the coefficient $T_{l t}^{*} / \tilde{m}$ is nondimensional. Once $\hat{u}$ is transformed into the same coordinate frame as the acceleration of the spacecraft relative to a body $P_{q}$, the additional acceleration term is straightforwardly added to the equations of motion. When modeling the motion of a spacecraft relative to the Moon within a Sun, Earth, and Moon point mass ephemeris model, the low-thrust-enabled equations of motion are written as:

$$
\begin{aligned}
X_{L, s / c}^{\prime \prime} & =-\frac{G M_{L}}{R_{L, s / c}^{3}} X_{L, s / c}+G \sum_{j=S, E} M_{j}\left(\frac{X_{s / c, j}}{R_{s / c, j}^{3}}-\frac{X_{L, j}}{R_{L, j}^{3}}\right)+\frac{T_{l t}^{*}}{m} u_{X} \\
Y_{L, s / c}^{\prime \prime} & =-\frac{G M_{L}}{R_{L, s / c}^{3}} Y_{L, s / c}+G \sum_{j=S, E} M_{j}\left(\frac{Y_{s / c, j}}{R_{s / c, j}^{3}}-\frac{Y_{L, j}}{R_{L, j}^{3}}\right)+\frac{T_{l t}^{*}}{m} u_{Y} \\
Z_{L, s / c}^{\prime \prime} & =-\frac{G M_{L}}{R_{L, s / c}^{3}} Z_{L, s / c}+G \sum_{j=S, E} M_{j}\left(\frac{Z_{s / c, j}}{R_{s / c, j}^{3}}-\frac{Z_{L, j}}{R_{L, j}^{3}}\right)+\frac{T_{l t}^{*}}{m} u_{Z}
\end{aligned}
$$

where the subscript $E$ identifies the Earth, $s / c$ identifies the spacecraft, $L$ corresponds to the Moon and $S$ indicates the Sun. Since these three equations of motion feature the time-dependent mass of the spacecraft, the 
mass flow rate equation is also required and must be simultaneously integrated along with the spacecraft state. Together, these differential equations are used to numerically integrate the augmented state of the spacecraft, $\bar{X}_{e}=[X, Y, Z, \dot{X}, \dot{Y}, \dot{Z}, m]$, where the nondimensional position and velocity components are expressed relative to the Moon in the Moon J2000 inertial coordinate system. At each epoch during the integration process, the position coordinates corresponding to each body, relative to a designated basepoint located at the Moon, are accessed using the Jet Propulsion Laboratory DE421 ephemerides via the SPICE toolkit. ${ }^{12,13,14}$ Relative position coordinates are then used to integrate the motion of a spacecraft relative to the Moon in the low-thrust-enabled four-body point mass ephemeris model. Alternatively, for motion predominantly in the Earth vicinity, the equations of motion can be written relative to the Earth for better performance during numerical integration.

\section{DIFFERENTIAL CORRECTIONS TO RECOVER AN END-TO-END TRAJECTORY}

To construct a continuous trajectory for a low-thrust-enabled spacecraft traveling within a point mass ephemeris model of the Earth, Moon and Sun, a variable-time multiple shooting algorithm is employed. ${ }^{10}$ The general procedure for a multiple shooting algorithm involves intermediate nodes, described by a set of free variables, that are simultaneously integrated to produce a sequence of arcs and, then, iteratively corrected to recover a continuous trajectory that satisfies a set of constraints. To demonstrate the formulation of the multiple shooting algorithm employed in this application, the conceptual representation in Figure 2 serves as a reference. In this illustration, the path of a spacecraft relative to a central body is sought as it travels under the gravitational influence of the central body and any additional perturbing bodies, as well as the action of a low-thrust engine.

Consider $n_{e}$ nodes, indicated via blue circles in Figure 2, to produce a sequence of arcs that, when integrated forward in time, form the initial guess for a trajectory. The $i$-th node is completely specified by the vector $\bar{s}_{i}=\left[X_{i}, Y_{i}, Z_{i}, \dot{X}_{i}, \dot{Y}_{i}, \dot{Z}_{i}, \tilde{m}_{i}, T_{\text {node }, i}, T_{i n t, i}\right]^{T}$. In particular, the description of each node includes: its nondimensional position, $\left(X_{i}, Y_{i}, Z_{i}\right)$, and velocity, $\left(\dot{X}_{i}, \dot{Y}_{i}, \dot{Z}_{i}\right)$, relative to body $P_{q}$ in a $P_{q}$-centered J2000 inertial frame; the spacecraft mass, $\tilde{m}_{i}$, to ensure mass continuity across arcs that employ low-thrust; the epoch at each node, $T_{n o d e, i}$, expressed relative to a fixed initial epoch and nondimensionalized via the characteristic time quantity, $t^{*}$, of an appropriate $P_{p}-P_{q}$ system; and the nondimensional integration time,

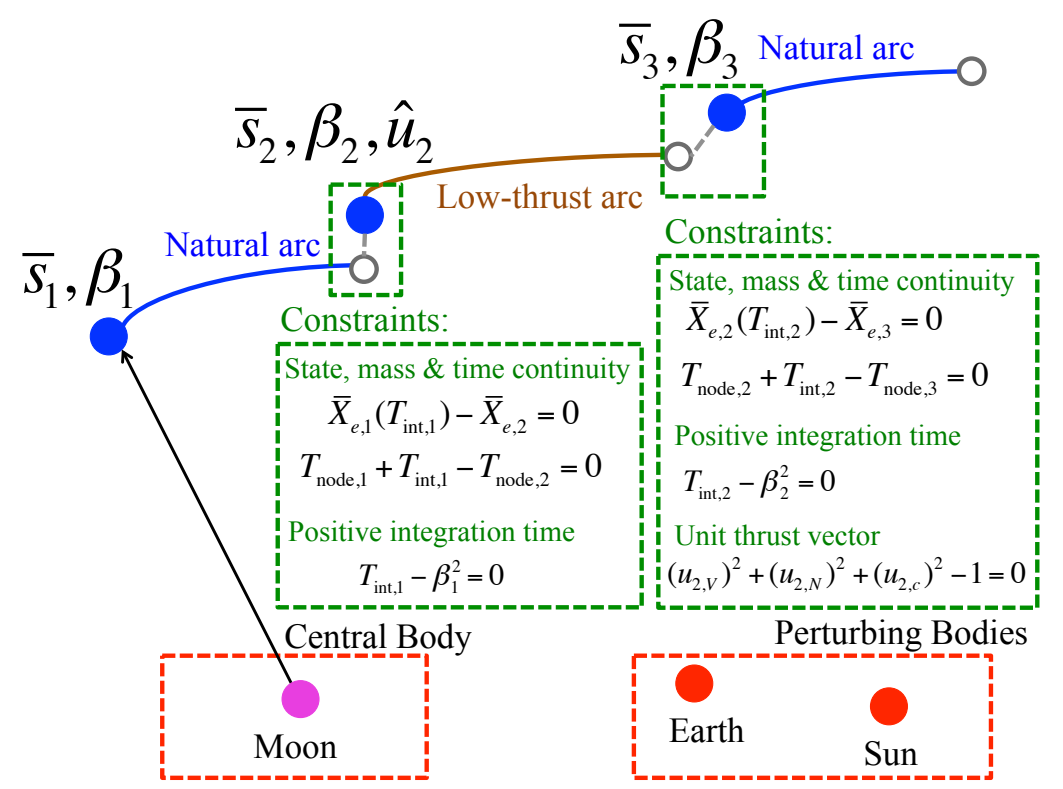

Figure 2: Illustration of variable time multiple shooting formulation to target a continuous trajectory for a low-thrust-enabled spacecraft in an ephemeris model. 
$T_{i n t, i}$. Together, these variables completely represent each node. However, to completely describe the arc associated with a given node, a constant binary parameter is used to distinguish between a natural arc, displayed in blue in Figure 2, and a low-thrust arc, indicated in brown. Although this parameter must be predefined, the integration time along an arc is allowed to vary towards zero, thereby enabling a low-thrust arc to be essentially eliminated, if necessary, to recover a continuous path. For a low-thrust-enabled arc, the three components of the unit thrust direction vector, $u_{V}, u_{N}$, and $u_{C}$, must be specified. To identify arcs over which the low-thrust engine is activated, as well as the basepoint body used to express the position and velocity vectors, constant vectors with integers encoding this information are employed. Using this description, each of the arcs along a trajectory is completely defined.

Multiple arcs, computed by integrating a set of nodes forward in time, are iteratively refined until they satisfy a sequence of constraints that produce a continuous trajectory that leverages both natural and low-thrustenabled motion. First, connectivity between two neighboring arcs requires state, mass and time continuity. In particular, the position, velocity and mass at the end of the $i$-th arc, integrated forward for a time $T_{i n t, i}$, must equal the position, velocity and mass at the $i+1$-th node to within an acceptable tolerance. In addition, since the ephemerides of the central and perturbing bodies are time-dependent, the epoch, $T_{n o d e, i}+T_{\text {int }, i}$, at the end of the $i$-th arc must equal the epoch, $T_{n o d e, i+1}$, at the $i+1$-th node; this condition is labeled a time continuity constraint. ${ }^{7}$ Furthermore, to ensure that the integration time along the $i$-th arc is positive, an inequality constraint is converted to an equality constraint that leverages a slack variable, $\beta_{i}$. This constraint is expressed in the form $T_{i n t, i}-\beta_{i}^{2}=0$. Then, for any low-thrust-enabled arcs, an additional constraint is required: the thrust vector components, $u_{V}, u_{N}$, and $u_{C}$, must produce a unit vector. Thus, the unit thrust vector constraint is written as $u_{V}^{2}+u_{N}^{2}+u_{C}^{2}-1=0$. The required constraints for the $i$-th segment are then summarized as follows:

$$
\begin{array}{ccc}
X_{i}\left(T_{i n t, i}\right)-X_{i+1}=0 & Y_{i}\left(T_{i n t, i}\right)-Y_{i+1}=0 & Z_{i}\left(T_{i n t, i}\right)-Z_{i+1}=0 \\
\dot{X}_{i}\left(T_{i n t, i}\right)-\dot{X}_{i+1}=0 & \dot{Y}_{i}\left(T_{i n t, i}\right)-\dot{Y}_{i+1}=0 & \dot{Z}_{i}\left(T_{i n t, i}\right)-\dot{Z}_{i+1}=0 \\
\tilde{m}_{i}\left(T_{i n t, i}\right)-\tilde{m}_{i+1}=0 & T_{\text {node }, i}+T_{\text {int }, i}-T_{\text {node }, i+1}=0 & T_{\text {int }, i}-\beta_{i}^{2}=0
\end{array}
$$

and over the segments for which the low-thrust engine is activated, $u_{V, i}^{2}+u_{N, i}^{2}+u_{C, i}^{2}-1=0$. Together, these constraints are simultaneously employed at each step of an iterative corrections algorithm to recover a continuous trajectory for a low-thrust-enabled spacecraft under the influence of a point mass ephemeris model of the Earth, Moon and Sun. ${ }^{10}$ In practice, additional constraints may need to be enforced at selected nodes along a trajectory. Such constraints, which may link to mission requirements or may be required for analysis, involve the epoch, spacecraft mass, inertial state components, apse conditions, energy via the Jacobi constant, rotating frame state components or orbital elements. When required, such constraints are added to the continuity, positive time and unit thrust vector constraints in the multiple shooting algorithm implemented within MATLAB, and their derivatives may be computed using any combination of analytical expressions and numerical approximations. ${ }^{10}$

\section{TRAJECTORY DESIGN FRAMEWORK}

By leveraging known natural dynamical structures that exist in a simplified, autonomous dynamical model, a rapid and well-informed process for constructing a trajectory for a low-thrust-enabled small satellite is demonstrated. In particular, the application of dynamical systems techniques enables the identification of known solutions that exist within a simplified natural gravitational environment. This insight offers a scheme to predict the bounds on the motion, while also supporting the construction of an initial guess for the trajectory prior to corrections in a operational-level modeling environment. ${ }^{4}$ The capability for a rapid and guided exploration of the trajectory design space is particularly beneficial for CubeSat missions that possess limited propulsive capability and, as a secondary payload, are subject to significant uncertainty in their deployment conditions. Furthermore, the construction of a framework for CubeSat trajectory design, as demonstrated for the Lunar IceCube mission, enables rapid redesign as the deployment and spacecraft hardware parameters evolve during both the development and operational phases of the mission. This framework involves decomposing the trajectory into three segments: 
- An Earth outbound segment, originating at deployment

- A phasing and energy adjustment segment that exploits solar gravity, and

- A lunar approach and capture portion.

To illustrate this trajectory sequence, consider a sample point solution, originally designed by David Folta at NASA GSFC for a thrust magnitude of $1.2 \mathrm{mN}$ and an earlier set of deployment conditions (from August 2015), as depicted in the top plot within Figure $3{ }^{4,11}$ In this figure, blue portions along the trajectory indicate natural motion while green curves correspond to arcs where the low-thrust engine is activated. In the three plots at the bottom of Figure 3, the arcs comprising each of the three segments are highlighted, while portions of this sample solution that are not included in the segment of interest are colored gray. Of these three segments along the trajectory sequence, the focus of this investigation is the phasing and energy adjustment arc, which exhibits the largest variability in terms of the available geometries and path characteristics. To explore and identify natural dynamical structures that may offer a pathway to link a high energy deployment state to a lunar capture arc, a Sun-Earth CR3BP model is leveraged, along with Poincaré mapping strategies. ${ }^{10}$ This framework is employed to demonstrate the construction of an initial guess for a new set of deployment conditions via dynamical systems techniques and insight. This initial guess trajectory is then corrected in a point mass ephemeris model that incorporates the additional acceleration of a low-thrust engine. The resulting trajectory supplies an initial guess for corrections and optimization within a higher fidelity dynamical model and, eventually, an operational-level modeling environment.
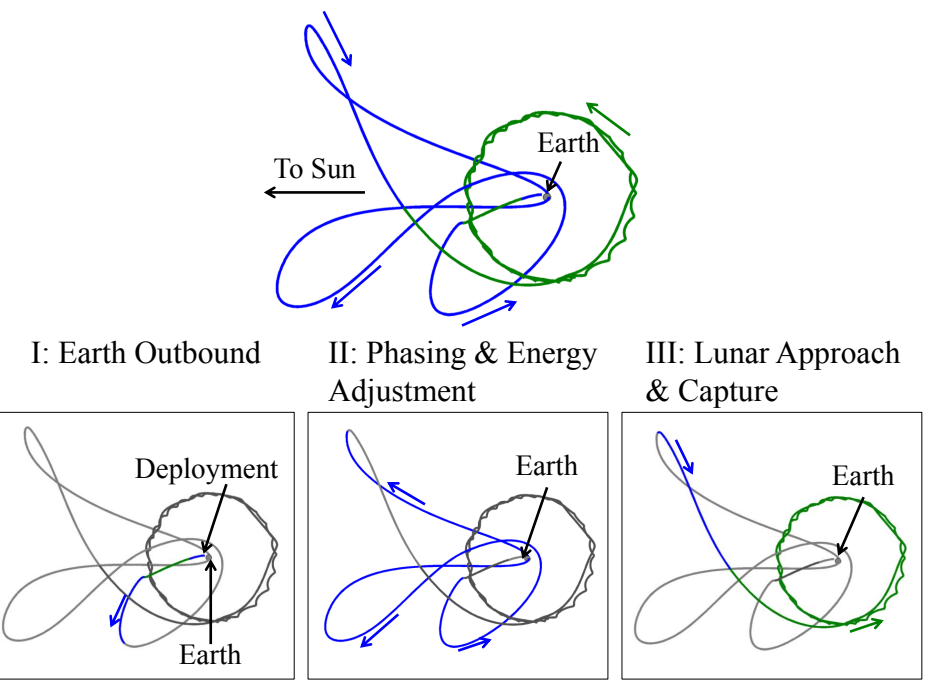

Figure 3: Illustration of the trajectory sequence employed in this investigation, demonstrated using a point solution for the original deployment conditions (top). Portions of the trajectory comprising each of three segments are isolated in the inset images (bottom).

\section{Earth Outbound Segment}

Given the original trajectory design, as illustrated in Figure 3, adapting to a new deployment state is nontrivial. For example, new deployment conditions are introduced and are current as of December 2016. Simulation of these most recently updated deployment conditions reveals that, naturally, the associated trajectory quickly departs the Earth vicinity. For a deployment epoch date of October 7, 2018, the initial state corresponds to a highly energetic path when integrated forward in time in a point mass ephemeris model and incorporating a lunar encounter. In fact, the associated path is depicted in nondimensional coordinates in Figure 4 in (a) the Sun-Earth rotating frame and (b) the Earth-Moon rotating frame, with zoomed-in views near the Earth appearing in the insets. In Figure 4(a), a circular approximation to the lunar orbit is overlaid 
(a) Sun-Earth Rotating Frame

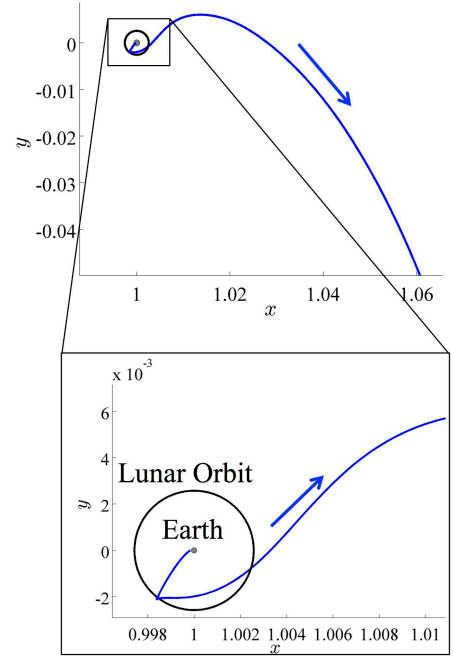

(b) Earth-Moon Rotating Frame

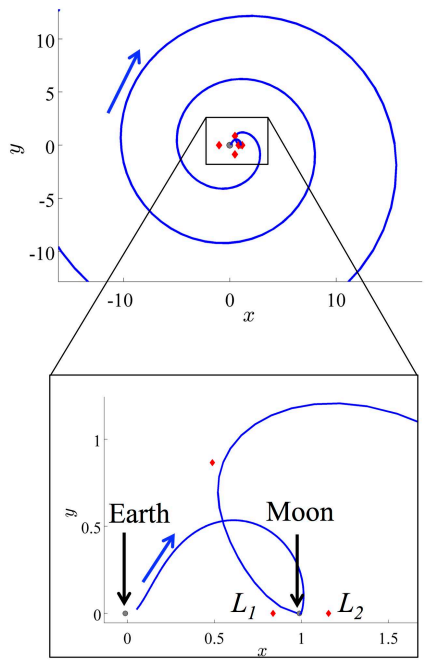

Figure 4: Natural motion corresponding to forward propagation of the recent Lunar IceCube deployment conditions over 200 days in nondimensional coordinates in (a) a Sun-Earth rotating frame and (b) an EarthMoon rotating frame.

in black while Figure 4(b) includes the location of the Earth and Moon as gray-filled circles and the libration points as red diamonds. As evident in both views represented within this figure, the spacecraft undergoes a trailing edge lunar flyby prior to quickly departing the Earth vicinity. Since the Lunar IceCube spacecraft must be delivered within a reasonable time interval to its final low altitude lunar orbit to commence scientific observations, propulsive intervention is required.

By introducing low-thrust arcs shortly after deployment, the flyby and post-flyby conditions are sufficiently altered to produce a trajectory that remains within the Earth vicinity. To demonstrate the impact of preperilune low-thrust arcs, consider the following operationally feasible arc sequence:

- An $8 \mathrm{hr}$ natural arc post-deployment to enable orbit determination,

- A $2 \mathrm{hr}$ low-thrust-enabled path with the thrust vector aligned with the velocity direction for thruster calibration,

- A $2 \mathrm{hr}$ natural arc for orbit determination and verification of the thruster performance,

- Three cycles of a $20 \mathrm{hr}$ low-thrust-enabled segment with the thrust vector aligned with the velocity direction to increase energy and raise the perilune followed by a $10 \mathrm{hr}$ natural arc for orbit determination,

- Coast until lunar periapsis, and

- A natural post-flyby arc until that continues until apogee.

For each low-thrust-enabled arc, a thrust magnitude of $0.9 \mathrm{mN}$ is employed for illustration. This sequence of arcs is depicted in Figure 5 in the (a) Sun-Earth rotating frame and (b) Earth-Moon rotating frame. In each figure, natural coasting segments are depicted in blue while low-thrust arcs are indicated in green. In Figure 5(a), a circular approximation to the lunar orbit is overlaid in black while Figure 5(b) includes the location of the Earth and Moon as gray circles and the libration points as red diamonds. The motion of the spacecraft is simulated until the first apogee following the lunar flyby. For this example, the apogee is prograde with respect to the Earth as viewed in the Sun-Earth rotating frame, i.e., the motion of the spacecraft is instantaneously counterclockwise. In fact, for this particular epoch and deployment state, the incorporation of three $20 \mathrm{hr}$ low-thrust arcs with a thrust vector aligned with the velocity direction and separated by coasting segments with a duration of $10 \mathrm{hrs}$ raises the lunar periapsis radius to $5080 \mathrm{~km}$ from approximately $2314 \mathrm{~km}$ 
(a) Sun-Earth Rotating Frame

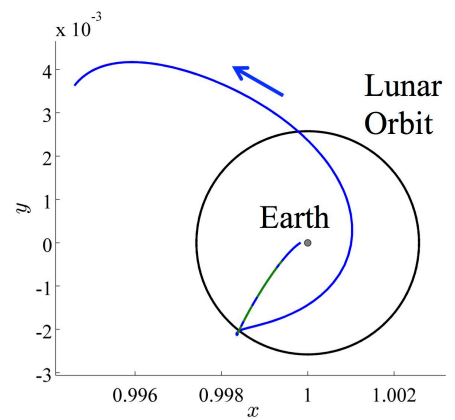

(b) Earth-Moon Rotating Frame

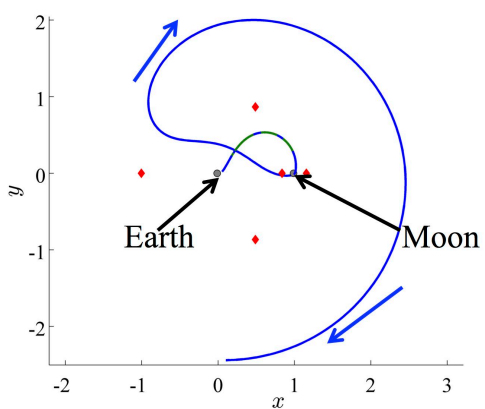

Figure 5: Path of Lunar IceCube from deployment to apogee, produced by leveraging both the low-thrust engine and a lunar flyby in (a) a Sun-Earth rotating frame and (b) an Earth-Moon rotating frame.

for the entirely natural trajectory depicted in Figure 4. Note that another deployment state and epoch may produce distinctly different behavior. ${ }^{4}$ Nevertheless, in addition to the reduction of speed provided by the given finite duration burn, raising the lunar periapsis reduces the acceleration boost supplied by the Moon and, therefore, the velocity of the spacecraft after the lunar flyby. As a result, an apogee occurs within the Earth vicinity and the path remains, at least temporarily, captured. This apogee is characterized and leveraged to link the Earth outbound portion of the transfer to the long phasing and energy adjustment segment.

By varying the thrust force direction and duration along each of the pre-flyby low-thrust arcs, the location and velocity of the first apogee along a temporarily captured path is significantly impacted. Assume the same trajectory sequence as in the previous example from Figure 5. While the deployment state is fixed, the direction of the thrust vector along each of the pre-perilune low-thrust-enabled arcs are varied, impacting the apogee conditions. To explore the variation in the state components at this apogee, consider a fixed burn duration of $20 \mathrm{hrs}$. Then, the thrust direction is varied up to 20 degrees relative to the velocity direction and is defined as constant as viewed in a Moon-centered VNC frame over each burn segment in a single simulation. The resulting trajectories are numerically integrated forward in a point mass ephemeris model from deployment to the first apogee; the parameters describing both the perilune and apogee are impacted. Figure 6 depicts the variation in these parameters for various thrust vector directions, where the post-deployment lunar flyby conditions are visualized on the b-plane in Figure 6(a) and (b), and the resulting apogees are displayed in a Sun-Earth rotating frame in Figure 6(c). In Figure 6(a), each b-plane coordinate describing the incoming flyby asymptote is colored by the Jacobi constant of the corresponding perilune state as computed in the Earth-Moon CR3BP and indicated via the colorbar. In Figure 6(b), each b-plane coordinate is colored by the Jacobi constant of the apogee as computed in the Sun-Earth CR3BP. Then, in Figure 6(c), each apogee is colored by the Jacobi constant at apogee, computed in a Sun-Earth CR3BP. Analysis of these figures reveals that pointing the thrust vector up to 20 degrees off the velocity direction can sufficiently reduce the energy along the transfer path to ensure temporarily captured motion in the Earth vicinity, while also shifting the intersection of the incoming asymptote and the b-plane by thousands of kilometers. Accordingly, the perilune radius can also be raised by as much as $2600 \mathrm{~km}$. This variation in the lunar flyby distance significantly impacts the radius and energy at the post-perilune apogee, as represented in Figure 6(c). In fact, the apogees displayed in Figure 6(c) vary by approximately 760,000 km in distance to the Earth and almost 90 degrees in their orientation as displayed in a Sun-Earth rotating frame. Furthermore, the Jacobi constant at these apogees possesses values between 3.000636 and 3.001185, which bound (above and below) the Jacobi constant value of $L_{2}$, indicating a large variety of the available flow structures in the vicinity of the Earth. Accordingly, there may be significant flexibility in the geometry and existence of transfers that return the spacecraft to the lunar vicinity. Such insight into the variation in the apogee condition is useful when linking the Earth outbound segment to the phasing and energy adjustment segment, as well as the identification of feasible transfer regions. 

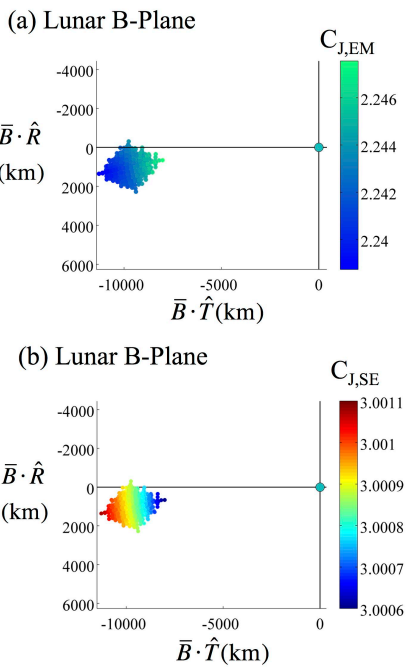

(c) Sun-Earth Rotating Frame

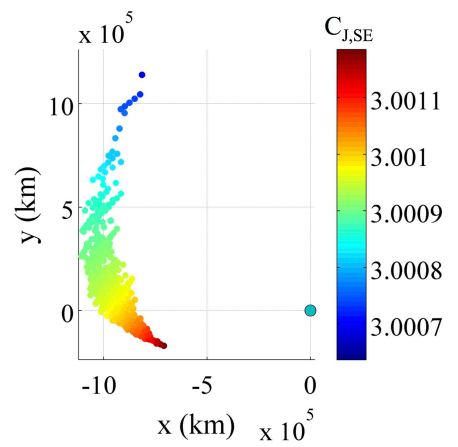

Figure 6: Over approximately 729 simulations, impact of varying thrust direction up to 20 degrees from the velocity direction on (a) lunar b-plane coordinates colored by the Earth-Moon Jacobi constant at perilune, (b) lunar b-plane coordinates colored by the Sun-Earth Jacobi constant at apogee, and (c) post-flyby apogee as viewed in the Sun-Earth rotating frame and colored by the Sun-Earth Jacobi constant at apogee.

\section{Phasing and Energy Adjustment Segment}

Following the post-deployment encounter with the Moon, one option for the Lunar IceCube trajectory design is leveraging the gravity of the Sun prior to approaching and capturing into the lunar science orbit. While this longest trajectory segment remains within the Earth vicinity, natural dynamical structures within the Sun-Earth system can be actively selected to modify both the energy and phasing of the spacecraft in its orbit. To reduce the number of deterministic thrusting arcs required along this portion of the Lunar IceCube mission trajectory, predominantly natural motions are preferred. Accordingly, Poincaré mapping techniques are useful in exploring the geometry of the natural flow corresponding to trajectories that persist within the Earth vicinity. These maps are constructed in the CR3BP, a model that offers a reasonable approximation to the dynamics in the true Sun-Earth system. Furthermore, these maps allow a prediction for the geometry of potentially successful transfers as constructed in this simplified dynamical model, as well as the corresponding regions of existence for transfers that link to a lunar capture arc in this model and serve as a design strategy in the ephemeris model.

To simplify the visualization of a large array of trajectories at a single energy level in the CR3BP, planar apoapsis maps are employed. Construction of these maps within the Sun-Earth CR3BP is demonstrated for a sample fixed value of the Jacobi constant, $C_{J}=3.0008813$. At this value of the Jacobi constant, both the $L_{1}$ and $L_{2}$ gateways are slightly open and only a small number of trajectories depart the Earth vicinity, enabling a clear demonstration of the analysis employed in this investigation. First, feasible initial conditions in the Earth vicinity are seeded in the rotating frame using nondimensional configuration space coordinates that lie within the zero velocity curves, and between the $L_{1}$ and $L_{2}$ gateways. These feasible sets of initial conditions are also represented as an apoapsis with respect to the Earth. For a state to be considered an apoapsis with respect to the Earth in the Sun-Earth rotating frame, it must possess a relative position vector $\bar{r}=[x-1+\mu, y, z]$, that is instantaneously perpendicular to the relative velocity vector, $\bar{v}=[\dot{x}, \dot{y}, \dot{z}]$, such that $\bar{r} \cdot \bar{v}=0$. Furthermore, the radial acceleration at an apoapsis must be negative. For this investigation, only planar motion is considered when creating apoapsis maps in the CR3BP. Although feasible trajectories within the true ephemeris model exist in three dimensional space, the out-of-plane component of motion along each sample transfer is small. Accordingly, planar motion in the CR3BP offers a valuable preliminary approximation with the added benefit of straightforward visualization. The direction of the velocity vector at each apoapsis is selected across the entire map such that the direction of motion is uniformly either prograde with respect to the Earth or 
retrograde, i.e., counter-clockwise or clockwise, respectively. Thus, for various combinations of the planar position components, the direction of the velocity is determined via orthogonality. For a specified value of the Jacobi constant, the unit vector along the velocity direction is then scaled using the velocity magnitude, computed as $v=\sqrt{2 U^{*}-C}$. Each initial apoapsis, seeded within the vicinity of the Earth, is then propagated forward for a specified number of revolutions about the Earth, from the perspective of the rotating frame. Initial conditions that produce trajectories that either impact the Earth or pass through the Sun-Earth $L_{1}$ or $L_{2}$ gateways are discarded. The remaining initial conditions are plotted in configuration space, producing a composite representation of the initial apoapses corresponding to trajectories that remain within the Earth vicinity as predicted by the Sun-Earth CR3BP. As an example, two apoapsis maps are displayed in Figure 7 for the Jacobi constant value $C_{J}=3.0008813$, representing trajectories that complete one revolution about the Earth without departing through the $L_{1}$ or $L_{2}$ gateways or impacting the Earth. In Figure 7(a),

(a)

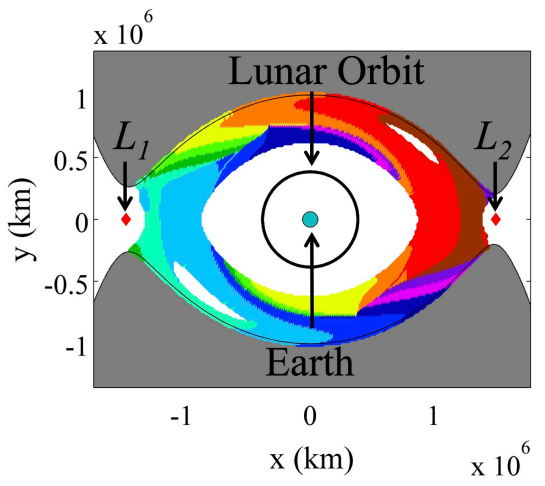

(b)

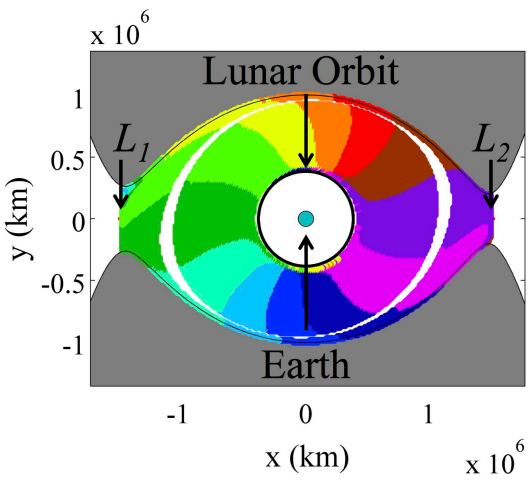

Figure 7: Apoapsis maps in the planar Sun-Earth CR3BP for trajectories that encircle the Earth once at $C_{J}=3.0008813$ for (a) prograde and (b) retrograde initial apoapsis conditions, colored by the angle of the final apoapsis after one revolution with the $+\mathrm{x}$ direction.

each initial apoapsis is prograde, while Figure 7(b) displays only retrograde apoapses. For convenience, these maps are represented in dimensional Earth-centered rotating coordinates. Gray shaded areas in each figure indicate forbidden regions, bound by the zero velocity curves, where motion cannot extend within the phase space of the CR3BP for the specified value of the Jacobi constant. Blue points locate apoapses that produce trajectories that remain within the Earth vicinity over one revolution and do not impact Earth. White regions, however, result in trajectories that do not fulfill these criteria. For prograde apses, these white regions are bound by the stable manifolds of the $L_{1}$ and $L_{2}$ Lyapunov orbits, while the white region in Figure 7(b) corresponds to trajectories that pass inside the radius of the Earth. ${ }^{10}$ Furthermore, red diamonds locate the equilibrium points, while a light blue circle indicates the location of the Earth and the black circle represents a circular approximation to the orbit of the Moon. Then, each initial apoapsis on these maps is colored by the angle of the final apoapsis after one revolution in the rotating frame relative to the Earth- $L_{2}$ line. A legend for this color scheme appears in Figure 8, with the Earth lo-

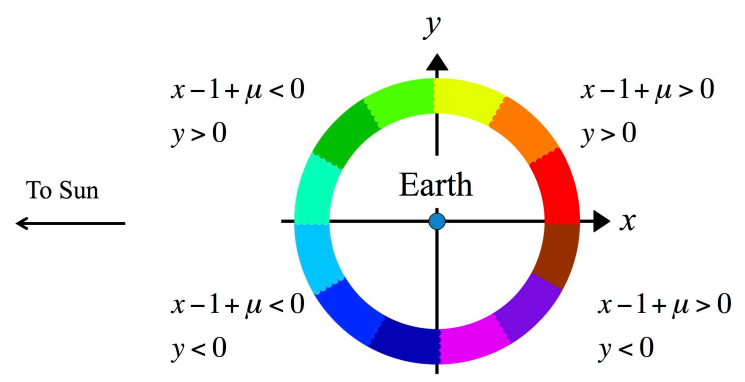

Figure 8: Color scheme used in the mapping strategy, indicating the angular location of the final apogee relative to the Sun- $L_{2}$ line. cated at the center as a blue-filled circle. To mitigate the effect of complicating the map visualization, the colors in this scheme vary every 30 degrees, indicating whether the final apse along a trajectory arc lies in the center of a quadrant, or close to a neighboring quadrant. For instance, initial conditions on an apoapsis map are colored teal, blue or dark blue if the final apse along 
the trajectory, propagated only for a specified number of revolutions around the Earth, possesses a state in the Sun-Earth rotating frame such that $x-1+\mu<0$ and $y<0$, i.e., it falls within the bottom left quadrant of the Earth-centered view. While this color scheme for the initial conditions on an apogee map does not capture the exact location of the final apses along the associated paths, it does enable rapid and straightforward evaluation of candidate transfers to identify those to be analyzed in further detail. The insight obtained through this application of Poincaré mapping strategies may guide the numerical targeting of outgoing lunar flyby conditions that subsequently deliver the Lunar IceCube spacecraft on a natural transfer path that requires little propulsive effort. For instance, white regions should not be targeted to avoid departing the Earth vicinity or impacting the Earth, while certain colored regions and their size may indicate the geometry of feasible transfers and their sensitivity to uncertainty in the initial conditions. In a chaotic and nonlinear system, simply integrating the finite set of post-deployment perilune conditions in Figure 6 forward for multiple Earth revolutions would yield only limited results that do not reflect the range of feasible transfer geometries, or supply insight into the corresponding regions of existence. However, mapping strategies supply a capability to visualize and rapidly identify natural candidate arcs in a simplified, yet representative, dynamical model. Such insight enables a well-informed process for constructing an initial guess for a trajectory that delivers the spacecraft from a highly energetic deployment state near the Earth to the vicinity of the Moon, as well as a rapid exploration of the trajectory design space.

\section{Lunar Capture Segment}

To locate arcs that approach the Moon and eventually result in captured motion, techniques from the construction of invariant manifolds are leveraged. ${ }^{4}$ Recall that the Lunar IceCube spacecraft is required to collect observations from a low altitude lunar orbit that is both elliptical and highly inclined. One strategy for reaching this orbit from a state that is located well beyond the lunar radius is based on the design of a trajectory that passes through the $L_{2}$ gateway with a Jacobi constant that is below the value corresponding to the $L_{2}$ libration point, i.e., $C_{J}\left(L_{2}\right)$. Then, the low-thrust engine is activated in a direction close to the anti-velocity vector, effectively reducing the spacecraft velocity. Simultaneously, with an active thrust force, the Jacobi constant increases, with the $L_{2}$ and, then, $L_{1}$ gateways closing, thereby bounding the motion of the spacecraft to the vicinity of the Moon and yielding captured motion. By modifying the thrust direction over a long time interval, the final science orbit is accessed. A significant challenge in designing a trajectory to deliver the spacecraft from a highly energetic deployment state to the final science orbit via this strategy is locating a trajectory that passes through the $L_{2}$ gateway. Since the Sun-Earth-Moon system is inherently nonlinear and chaotic, an uninformed search for trajectories that achieve this goal may be cumbersome, tedious and unsuccessful. However, techniques from manifold computation approaches are useful: a desired lunar orbit is discretized and each fixed point is numerically integrated backwards in time in a point mass ephemeris model, with the thrust vector directed in the anti-velocity direction. ${ }^{4}$ In this investigation, to construct a successful lunar capture, one of the significant hurdles in trajectory design for the Lunar IceCube spacecraft is identifying trajectories that deliver the spacecraft to the lunar vicinity with a Jacobi constant value above that of $L_{1}$ and $L_{2}$. To achieve this goal, it is necessary to construct an initial guess for a lunar capture arc that completes multiple nearly-polar revolutions around the Moon. Higher order gravitational models, which significantly influence motion close to the bodies, can be then be employed in additional analyses to exactly target motion that subsequently reaches the low lunar orbit.

To explore the dynamical flow toward the destination orbit, a desirable lunar orbit is defined and integrated in negative time with the low-thrust engine activated. The desired science orbit, with a Jacobi constant above that of $L_{1}$ and $L_{2}$, is described by a semi-major axis of $4,287 \mathrm{~km}$, an eccentricity of 0.5714 , an inclination of approximately 90 degrees, and a predefined final epoch. The right ascension of this orbit is sampled within the range $\Omega=[0,360)$. States with true anomalies in the range $\theta^{*}=[0,360)$, are sampled along this orbit and integrated backwards in time in a point mass ephemeris model with the thruster activated until reaching the Jacobi constant of $L_{1}$. With the thruster still activated, the trajectory is integrated further until the subsequent apse (periapsis or apoapsis). Departing from this state, the thruster is off and the spacecraft is naturally propagated further backwards in time until it reaches the $L_{1}$ or $L_{2}$ gateways, impacts the Earth or until reaching a maximum integration time. The parameters describing this trajectory are stored if they pass through the $L_{2}$ gateway and encounter an apogee beyond the lunar radius within a reasonable time frame. 
This process is then repeated by integrating backwards in time with the low-thrust engine activated to the next apse until the trajectory passes below the Earth radius or departs the Earth vicinity. A sample trajectory sequence is displayed in Figure 9 for the prescribed values of $a, e$, and $i$, a final epoch of 28601 MJD, a final mass of $13.5 \mathrm{~kg}$, a RAAN of 150 degrees and a true anomaly of 135 degrees. This sample trajectory is visualized in (a) a Moon-centered inertial J2000 equatorial frame and (b) a Moon-centered Earth-Moon rotating frame. In both plots, green indicates thrusting segments while blue curves identify natural coasting arcs. To generate a set of trajectories that may be used to construct a viable initial guess, a final spacecraft mass at arrival to the science orbit must be set. Since this quantity is not known a priori, it is estimated iteratively using the average thrust time for the spacecraft to depart the $L_{2}$ gateway. This process, i.e., generating a set of sample capture arcs, is demonstrated for the selected science orbit and a final epoch of 28695.0 MJD, with values for $\Omega$ and $\theta^{*}$ each varied in increments of 45 degrees, respectively. The final spacecraft mass is estimated to be equal to $13.5 \mathrm{~kg}$. Trajectories that experience an apogee within 250 days of passing through the $L_{2}$ gateway are stored and plotted in Figure 10(a) in the Sun-Earth rotating frame. Then, the recorded apogee for each of the stored trajectories is indicated by a circle colored by the epoch in terms of modified Julian date in Figure 10(a). These trajectories exhibit a variety of characteristics and possess apogees that occur in various regions of the phase space. To aid visualization and comparison to the phasing and energy adjustment segment, these apogees are also depicted on an apoapsis map in Figure 10(b).

(a) Moon-Centered Inertial J2000 Frame

(b) Moon-Centered Earth-Moon Rotating Frame
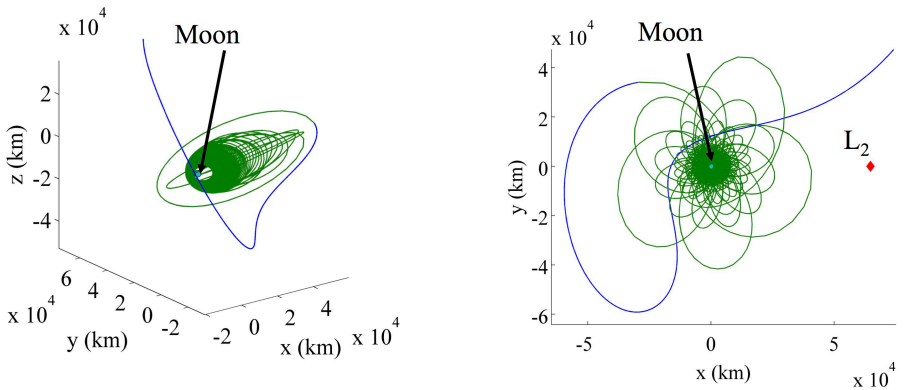

Figure 9: Sample arc that captures into the desired lunar science orbit, displayed in (a) a Moon-centered inertial J2000 equatorial frame, and (b) a Moon-centered Earth-Moon rotating frame. Green arcs indicate that the thruster is activated in the anti-velocity direction, while blue arcs correspond to natural motion

(a)

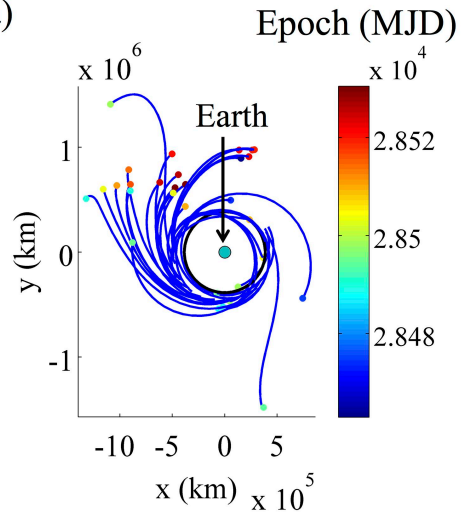

(b)

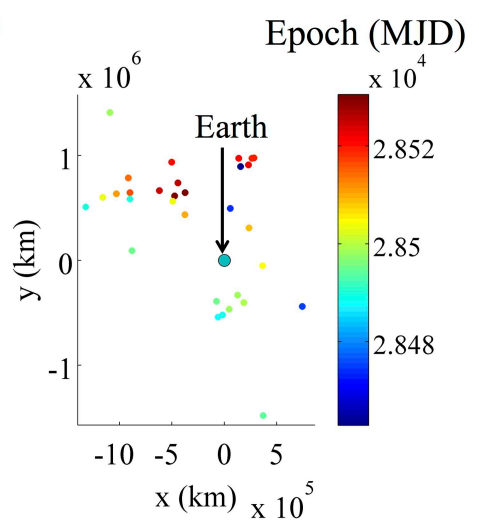

Figure 10: (a) Trajectories that deliver the spacecraft to the lunar vicinity as viewed in configuration space in the Sun-Earth rotating frame and (b) representations of these paths on an apoapsis map colored by the apogee epoch in MJD. 
Each apogee, represented by a planar projection of the configuration space variables in the Sun-Earth rotating frame, is colored by its corresponding epoch to identify lunar approach arcs that originate at a time similar to the time associated with the final state along the previous segment. Of course, this map does not represent all parameters describing the apogees within this set. However, this reduced dimension mapping does enable a rapid and straightforward evaluation of the trade space. From this set of apogees, candidate paths that deliver the spacecraft to the lunar vicinity are straightforwardly identified and incorporated into the construction of an initial guess for a complete end-to-end trajectory design.

\section{Sample Trajectory Construction}

Arcs comprising each segment of the transfer sequence are selected using apoapsis maps to construct an initial guess for a path that delivers the spacecraft from an initial deployment state to the lunar vicinity and is then corrected in a low-thrust-enabled point mass ephemeris model. This process is demonstrated for a single transfer using the most recent deployment information (current as of December 2016). As the deployment state and epoch continues to evolve throughout the EM-1 mission planning phase, the transfer sequence and Poincaré map definition may require updates; however, the general framework, constructed and demonstrated in this investigation, remains useful.

An Earth outbound transfer arc influences the potential geometries for feasible transfers in the phasing and energy adjustment segment. For this example, a single sequence of coasting and thrusting arcs is selected using a cyan point from Figure 6. Consider a sample trajectory generated in a point mass ephemeris model of the Earth, Moon and Sun and plotted as a planar projection in Sun-Earth rotating coordinates in Figure 11. Recall that blue arcs identify natural motion while green arcs indicate that the $0.9 \mathrm{mN}$ low-thrust engine is activated and a black circle approximates the lunar orbit. The final apogee along this transfer segment is prograde when viewed in the rotating frame, possessing a Jacobi constant value of approximately $C_{J}=3.000854$ and an epoch of 28436.5 MJD. Furthermore, at the end of this segment, the spacecraft mass is equal to $13.99 \mathrm{~kg}$. Each of these characteristics significantly impact the construction of the remainder of the trajectory.

To identify a feasible path for the energy and phasing adjustment segment, the Poincaré mapping strategy is employed. For this example, consider transfer arcs that encircle the Earth once, essentially reducing the time of flight along this portion of the path. An apoapsis map is then constructed for this type of transfer at $C_{J}=3.000854$ with prograde initial conditions, matching the Ja-

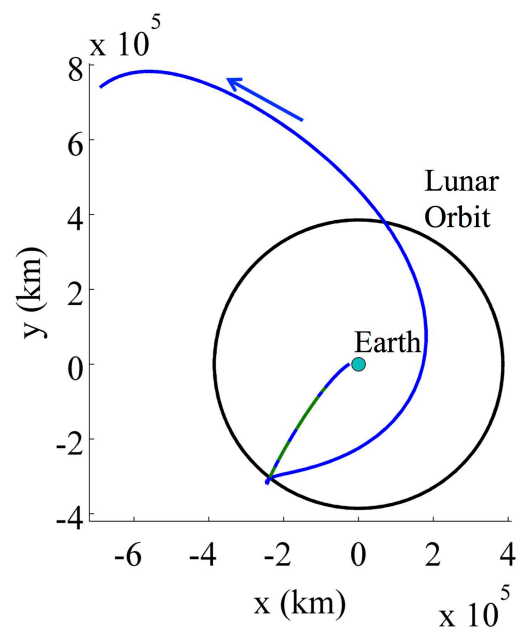

Figure 11: Planar projection of the selected outbound segment in Earthcentered rotating coordinates in the Sun-Earth frame. cobi constant value and the direction of motion at the apogee that designates the end of the Earth outbound segment. The resulting map is displayed in Figure 12. Recall that initial apogees that produce planar trajectories encircling the Earth once without departing through the $L_{1}$ or $L_{2}$ gateways or impacting the Earth are plotted on this map in Earth-centered rotating coordinates. Each initial condition is colored, according to the legend displayed on the right of Figure 12, by its fate after a subsequent revolution, represented by the angle of the final apoapsis state along the trajectory relative to the Sun- $L_{2}$ line. In addition, a circular approximation to the lunar orbit is plotted in black and the Earth is located by a gray-filled circle; the equilibrium points are plotted as red diamonds. Then, forbidden regions are again indicated by gray shaded regions. Overlaid on this apoapsis map is the location of the apogee state at the end of the Earth outbound segment, plotted as a gray star. Recall that the Earth outbound transfer arc is computed in a three-dimensional point mass ephemeris model, while the trajectories in this energy and phasing adjustment segment are propagated in a planar Sun-Earth CR3BP model. Thus, an exact match is not expected between the initial condition associated with the actual transfer arc in this segment and the end of the Earth outbound segment. However, the CR3BP does supply a reason- 


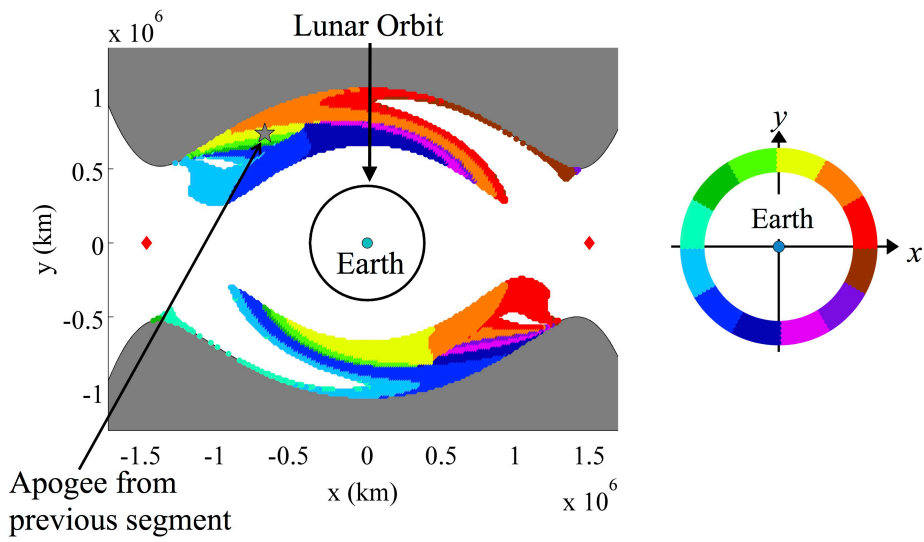

Figure 12: Apoapsis map in the planar Sun-Earth CR3BP for trajectories that encircle the Earth once at $C_{J}=3.000854$ for prograde initial conditions, colored by the angular location of the final apoapsis as indicated in the legend. The final apogee along the Earth outbound segment is indicated by a gray star.

able approximation to the true dynamics in the Sun-Earth system. Selecting an initial apogee near the final apogee along the previous segment should mitigate any necessity for a long thrusting arc to connect the two segments, while still providing sufficient flexibility to select the desired transfer geometry. Thus, this initial condition could lie in either a yellow, green or orange region, each of which yield transfers that possess a final apogee near the $+y$-axis. In this example, the location of the final apoapsis along this transfer arc offers a rapid assessment of the approximate geometry for the transfer. This coloring scheme is also useful if the epoch at which the spacecraft must reach the final science orbit is constrained. An initial condition in the yellow region near the gray star is selected close to the boundary of the orange area and propagated in the CR3BP to produce a planar trajectory that is plotted in the Sun-Earth rotating frame in Figure 13(a). This transfer, with a time of flight equal to 83.3 days, is then discretized into 20 subarcs, equally spaced in time. The spacecraft state at the beginning of each arc, as computed in the CR3BP, is then propagated forward in the point mass ephemeris model of the Sun, Earth and Moon. In support of this simulation, an initial epoch, equal to the epoch at the end of the previous segment, 28436.5 MJD, is assumed. As an estimate, the final apogee at the end of this transfer arc is approximated as 83.3 days following the initial epoch, i.e., 28519.7 MJD. Note that, since only natural motion is leveraged along this segment, the spacecraft mass remains unchanged at the value of $13.99 \mathrm{~kg}$. The resulting sequence of arcs is plotted in planar configuration space in Figure 13(b) with the nodes at the beginning of each segment located with green circles. Of course, each
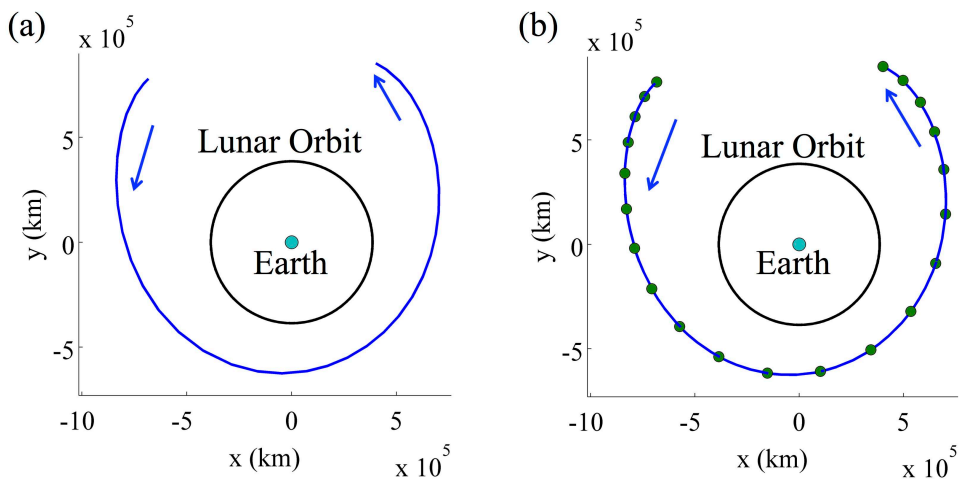

Figure 13: Selected transfer arc for energy and phasing adjustment segment plotted in the Sun-Earth rotating frame: (a) propagated in the CR3BP and (b) discretized and integrated in the point mass ephemeris model. 
of these arcs is three-dimensional and no longer produces a single continuous path. However, these nodes serve as an initial guess for the Sun-Earth segment along the complete transfer, one that is later adjusted in a corrections algorithm. Additionally, the map in Figure 13 supplies insight into the available geometries of candidate arcs for the Sun-Earth segment. For instance, the thin colored regions in the vicinity of the gray star in Figure 12 indicate that the location of the final apogee along the Sun-Earth segment of the transfer may be sensitive to the initial apogee state: such sensitivity may be beneficial in navigating back to a reference science orbit approach path with minimal propellant usage following small uncertainties in the deployment information, thruster performance or flyby conditions. However, a sufficiently large uncertainty that places the initial apogee close to the small white region in Figure 12, embedded within the light blue structure in the top left of the figure, may necessitate the use of long thrust arcs to ensure that the trajectory does not depart the Earth vicinity or impact the Earth.

A final transfer arc is, concurrently, selected to deliver the spacecraft to the lunar vicinity. Recall that the transfer arc selected in the energy and phasing adjustment segment produces a final apogee that is shifted to the $L_{2}$ side of the Earth in a Sun-Earth rotating frame. Thus, lunar approach arcs that possess an initial apogee on the $L_{2}$ side of the Earth in the Sun-Earth system with an epoch close to 28519.7 MJD are sought. Although a close match in energy between the beginning of the lunar approach segment and end of the phasing and energy adjustment segment is sought, the value of $C_{J}$ is no longer constant in the ephemeris model, especially with the incorporation of an additional gravitational contribution. Thus, a prediction of the value of $C_{J}$ at the end of the energy and phasing adjustment segment from the CR3BP may not be truly reflective of the actual value when corrected in an ephemeris model that also incorporates lunar gravity. Accordingly, the Jacobi constant is only loosely employed as a filter for selecting the apogee that defines the lunar approach arc. Alternatively, the epoch, location and direction of motion are incorporated as the dominant selection parameters. To produce an apoapsis map for identifying a lunar approach arc, the process outlined earlier is employed along with the specified science orbit. An appropriate final epoch at the science orbit is iteratively determined and set equal to $28693 \mathrm{MJD}$, while the right ascension for the orbit and the true anomaly for discretization are sampled within the range $[0,360)$ in increments of 40 and 10 degrees, respectively. The final spacecraft mass is set to $13.5 \mathrm{~kg}$. The resulting trajectories, in particular, those that pass through the $L_{2}$ gateway in backwards time, are stored and plotted in Figure 14(a) and (b) in the Earth-centered Sun-Earth rotating coordinates. In Figure 14(a), each apogee is colored by the number of days past the desired epoch, i.e., the epoch at the end of the previous transfer segment and equal to 28519.7 MJD, while in Figure 14(b), each apogee is colored by the Jacobi constant as computed in a Sun-Earth CR3BP. Overlaid on these plots is a gray star which locates the final apogee of the previous arc. Since arcs selected from this map should possess a position, epoch and energy similar to those of the reference apogee, a lunar approach apogee close to the gray star and in the red region of Figure 14(a) is preferred. This apogee should also possess a Jacobi constant close to the value corresponding to the final apogee along the Sun-Earth segment, i.e. $C=3.000854$, thereby also lying in a light blue to green region of Figure 14(b). If no apogees are detected in the desired regions of the
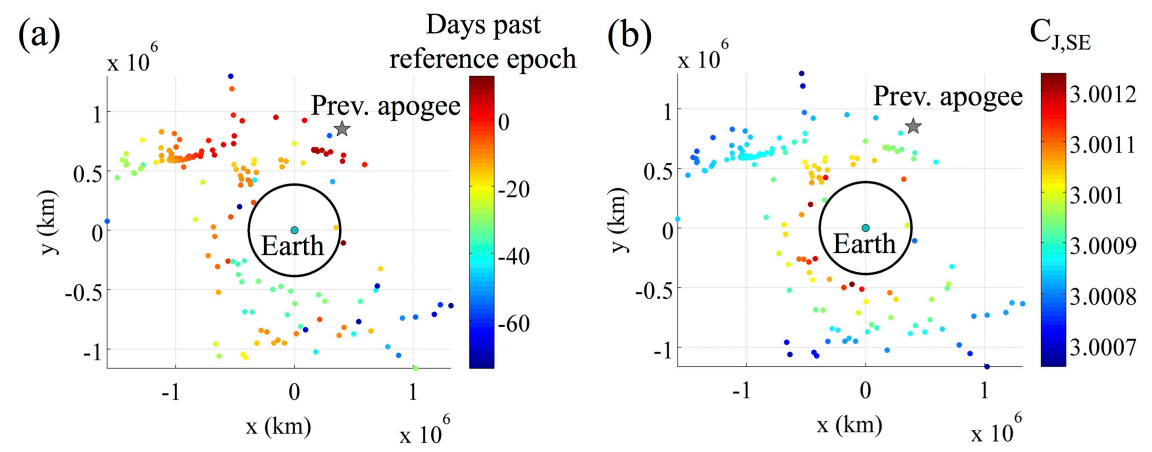

Figure 14: Apoapsis map representing trajectories that approach the lunar science orbit, projected into planar configuration space and colored by the epoch at each apogee. 
apoapsis maps near the final apogee along the previous segment, the final epoch can be iteratively modified and a new map constructed. Of course, the difference between the parameters describing the apogee at the end of the phasing and energy adjustment segment and the apogee at the beginning of the lunar approach arc impact the quality of the initial guess and, therefore, the potential for the differential corrections process to identify a continuous solution. Fortunately, in the current example, candidate apogees lie within desirable areas near the gray star. Thus, this map is employed to define a small range of RAAN and true anomaly values and iteratively recomputed to identify a good candidate arc: one corresponding to a final science orbit epoch of 28693.01 MJD, RAAN = $199.2 \mathrm{deg}$, TA = $68 \mathrm{deg}$. A lunar approach arc is selected in this vicinity, producing the trajectory plotted in Figure 15 and viewed in: (a) an Earth-centered view in the Sun-Earth rotating frame, (b) a planar zoomed-in view along the final portion of the transfer in the Moon-centered Earth-Moon rotating frame, and (c) a three-dimensional view of the final portion of the transfer in the Mooncentered Earth-Moon rotating frame. Along the trajectory in Figure 15, green segments indicate low-thrust arcs, while a blue line identifies a natural arc. Using this figure, the selected initial condition occurs at an epoch equal to 28523.6 MJD with a Jacobi constant value $C_{J}=3.000917$ and a spacecraft mass equal to $13.92 \mathrm{~kg}$; this mass is approximately $0.07 \mathrm{~kg}$ below the spacecraft mass at the end of the previous transfer segment and the epoch is discontinuous by approximately 4 days. In addition, the incoming lunar capture arc exhibits a lunar flyby distance of $100 \mathrm{~km}$ prior to activating the low-thrust engine. Of course, these parameters are adjusted during the iterations in a corrections algorithm, with short low thrust arcs introduced to assist in bridging the discontinuities. Since the goal of this investigation is the construction of a transfer that delivers the spacecraft to the lunar vicinity, the selected trajectory is clipped to include only a few revolutions of the Moon, rather than the entire lunar approach arc. In fact, these revolutions around the Moon serve to guide the corrector to a trajectory that reaches the lunar vicinity without the extra computational time associated with correcting a longer path. The exact trajectory to the final lunar science orbit is more appropriately identified in subsequent analyses that incorporate higher fidelity models to also capture perturbations from lunar gravitational harmonics.

Each of the arcs selected via dynamical systems techniques enables the construction of an initial guess for a trajectory that is ultimately corrected in a low-thrust-enabled point mass ephemeris model. In particular, each of the three segments depicted in Figures 11, 13 and 15 are concatenated chronologically

(a)

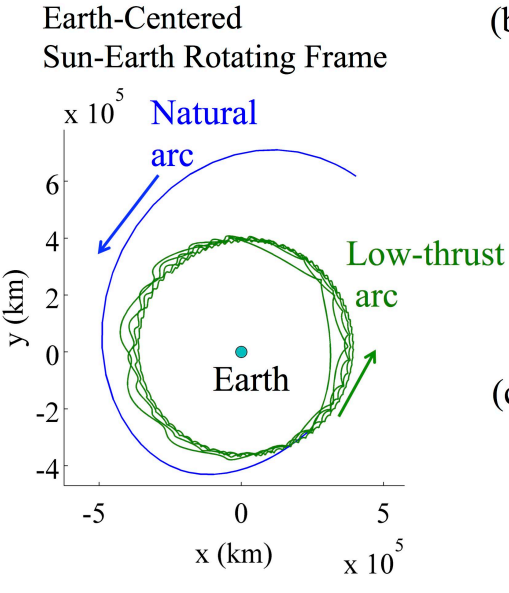

Moon-Centered

(b)
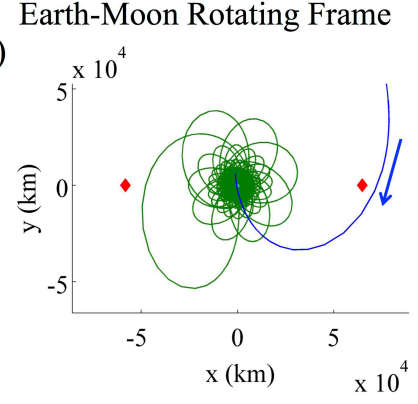

(c)

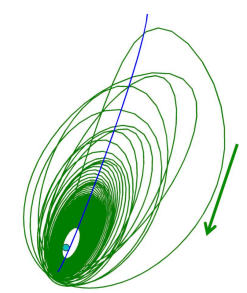

Figure 15: Selected trajectory that approaches the lunar vicinity: (a) a view of the entire trajectory in an Earth-centered Sun-Earth rotating frame, (b) zoomed-in planar view of the final portion in a Moon-centered Earth-Moon rotating frame and (c) a three-dimensional view in the Earth-Moon rotating frame. 
with short low thrust arcs introduced between each segment, i.e., the Earth outbound segment is followed by a short low thrust arc to connect to the energy and phasing adjustment segment, and then an additional low thrust path is followed by the lunar approach arc. Recall that the corrections algorithm employed in this investigation is formulated for simultaneous adjustment of a sequence of nodes described in terms of the body-centered position and velocity coordinates in a J2000 inertial frame, nondimensionalized by the characteristic quantities corresponding to the Sun-Earth or Earth-Moon system, as appropriate. Additional information that is recorded at each node includes the nondimensional time past a reference epoch (e.g., the deployment epoch), the integration time along the arc of interest as well as the spacecraft mass. For low-thrust-enabled arcs, the three components of the thrust direction unit vector are also stored as variables. To convert the initial guess in Figure 16 into a sequence of nodes, natural and low-thrustenabled segments are first separated. Then, each segment is discretized by a user-defined number of nodes that are equally spaced in time. For this sample trajectory, each of the states along the discretized initial guess is defined relative to the Moon. In addition, three constant vectors supply the corrections algorithm with information that is vital to the numerical integration process and evaluating continuity across all arcs, as well as accurately incorporating the low-thrust engine when appropriate. Specifically, these vectors reflect activation of the low-thrust engine, the basepoint body used

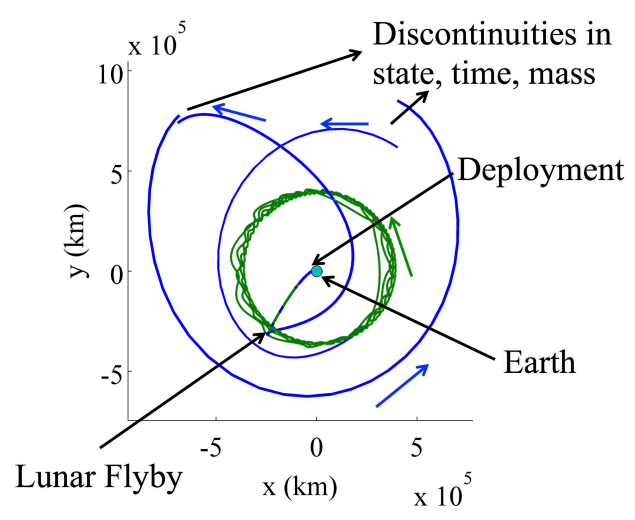

Figure 16: Discontinuous initial guess for a complete trajectory, plotted in Earth-centered Sun-Earth rotating coordinates. for defining the VNC unit vectors describing the thrust direction, and the body used as a basepoint for defining the state information. This initial guess is displayed in Earth-centered Sun-Earth rotating coordinates in Figure 16. Recall that green arcs locate low-thrust-enabled segments while blue indicates natural motion. Although the free variables representing each state are formulated in the inertial frame when inputted to the corrections algorithm, the Sun-Earth rotating view offers an intuitive representation for verification of the construction of the initial guess. Note that this initial guess exhibits clear discontinuities in the state, time and mass variables between each phase of the trajectory. However, short low-thrust arcs are added at these discontinuities to support the identification and computation of a continuous path. While selecting different arcs may reduce these discontinuities, this initial guess serves as an interesting example for demonstration purposes.

The constructed initial guess is supplied to a corrections algorithm that leverages a low-thrust-enabled point mass ephemeris model. Since this complete trajectory is comprised of arcs that pass close to each of the primaries as well as those that exist beyond the lunar radius, numerical issues due to increased sensitivity and the presence of significant discontinuities may appear. To mitigate such problems, a modified Newton's method is employed to update the free variable vector. Recall that the update equation for a Newton's method is formulated under the assumption that the current guess for a variable is close to the true solution. However, for an initial state that is not sufficiently close to a true solution for the constraint vector, the Newton strategy may diverge. Thus, an attenuation factor, $\gamma$, is introduced to the update equation for an underdetermined system such that, at each step, the free variable vector is iteratively modified as follows:

$$
\bar{V}^{i+1}=\bar{V}^{i}-\gamma D \bar{F}\left(\bar{V}^{i}\right)^{T}\left[D \bar{F}\left(\bar{V}^{i}\right) \cdot D \bar{F}\left(\bar{V}^{i}\right)^{T}\right]^{-1} \bar{F}\left(\bar{V}^{i}\right)
$$

for a free variable vector at the $i$-th iteration, $\bar{V}^{i}$, a constraint vector, $\bar{F}$, and a rectangular Jacobian matrix, $D \bar{F}$. Note that $\gamma$ can be assigned any value between 0 and 1 . Once the magnitude of the constraint vector falls below a threshold value, the update equation reverts to the traditional Newton's method, i.e., $\gamma=1$. The exact value for both $\gamma$ and the threshold on the constraint vector magnitude may be tuned by the user as the performance of the algorithm is evaluated. Alternative methods to eliminate any issues associated with ill-conditioning would serve as an interesting avenue of investigation in future analyses.

Corrections on the initial guess in Figure 16 enable the detection of a continuous trajectory in a low-thrustenabled ephemeris model that follows the constructed itinerary and delivers the spacecraft from a highly 
energetic deployment state to the lunar vicinity. The state, time and mass continuity constraints, as well as the positive integration time and unit thrust vector direction constraints, are simultaneously applied in a corrections algorithm with updates implemented until the trajectory is continuous to within a nondimensional tolerance on the constraint vector equal to $10^{-10}$. Following the completion of this iterative process, a corrected trajectory is identified. Additional constraints on the initial condition along the path are also enforced to match the specified deployment conditions. This solution is displayed in Figure 17 in (a) Earth-centered Sun-Earth rotating coordinates, (b) a zoomed-in view of the lunar vicinity in Moon-centered Earth-Moon rotating coordinates, (c) Earth-centered J2000 equatorial inertial coordinates and (d) Moon-centered J2000 equatorial inertial coordinates. Recall that green lines indicate low-thrust-enabled arcs while blue curves indicate natural motion. Comparison to the initial guess as constructed in Figure 16 reveals that the general geometry of the transfer is retained, demonstrating the value of leveraging natural dynamical structures within multi-body systems in the construction of a complex path for a low-thrust-enabled CubeSat. Although the final portion of the path does not capture the entire lunar approach path, the goal of this investigation is certainly achieved: recovery of a path that delivers the spacecraft from a highly energetic deployment state to the lunar vicinity, while maintaining a geometry consistent with the constructed initial guess. Identification of a trajectory that eventually encircles the Moon, with a sufficiently high inclination and an energy at which the $L_{1}$ and $L_{2}$ gateways are closed, addresses the most significant challenges in the construction of an initial guess for a feasible end-to-end transfer. Additional analysis in a higher-fidelity model that more accurately reflects the lunar gravitational field can be leveraged to identify subsequent low-thrust-enabled arcs that reach the desired lunar science orbit. Furthermore, corrections and optimization processes may be applied to this solution to reduce the total thrusting time and, therefore, propellant usage. This process may be used in future analyses to rapidly produce a variety of transfers, supporting an exploration of the available transfer geometries and corresponding regions of existence. ${ }^{10}$

(a)

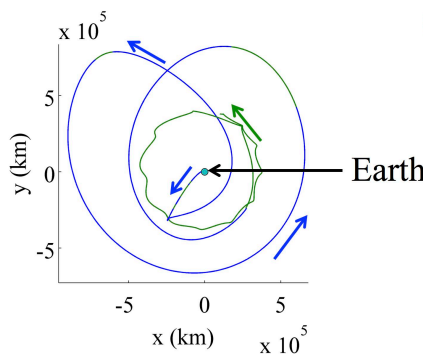

(c)

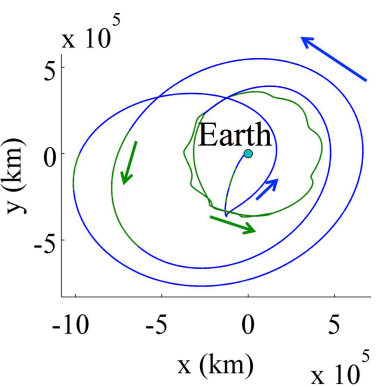

(b)

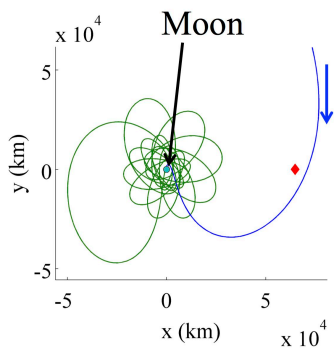

(d)

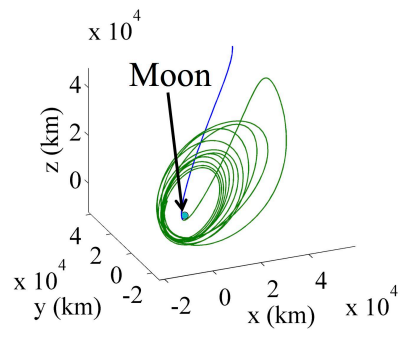

Figure 17: Continuous sample trajectory (a) plotted in Earth-centered Sun-Earth rotating coordinates, (b) a zoomed-in view in Moon-centered Earth-Moon rotating coordinates, (c) Earth-centered J2000 inertial coordinates and (d) Moon-centered J2000 inertial coordinates.

\section{CONCLUDING REMARKS}

The insight gained throughout this investigation demonstrates that a trajectory design framework, constructed using dynamical systems techniques, is valuable in designing a complex path for a spacecraft with limited propulsive capability within the chaotic gravitational environment of the Earth, Moon and Sun. The 
continuous solution constructed here serves as a suitable initial guess for corrections to a complete end-to-end path in an operational-level modeling environment and, potentially, an optimization algorithm. Of course, the exact transfer sequence and map definition may require updates as the deployment state frequently evolves throughout development of the mission. However, the framework developed in this investigation remains useful for constructing trajectories for a small spacecraft that must be delivered from a high energy deployment state near the Earth to a low lunar orbit. In fact, the design framework enables rapid redesign of this trajectory as the deployment information or spacecraft hardware specifications evolve by mitigating the challenges associated with searching for a point solution in a chaotic dynamical regime, while also supporting the exploration of a large and complex design space.

\section{ACKNOWLEDGMENT}

This work was completed at NASA Goddard Space Flight Center and Purdue University under NASA Grants NNX13AM17G and NNX16AT688A. The authors wish to thank Purdue University's School of Aeronautics and Astronautics and College of Engineering for their support, and the Zonta International Amelia Earhart Fellowship.

\section{REFERENCES}

[1] Asteroid Impact Mission, Accessed June 2016: http://www.esa.int/Our_Activities/ Space_Engineering_Technology/Asteroid_Impact_Mission/Asteroid_ Impact_Mission2

[2] P.E. Clark, B. Malphrus, R. MacDowall, D. Folta, A. Mandell, C. Brambora, D. Patel, S. Banks, K. Hohman, V. Hruby, K. Brown, J. Kruth, R. Cox, "Lunar Ice Cube: Determining Volatile Systematics Via Lunar Orbiting Cubesat," European Planetary Science Congress Vol. 10, 2015.

[3] Mars Cube One (MarCO), Accessed June 2016: http://www.jpl.nasa.gov/cubesat/ $\mathrm{missions/marco} \cdot \mathrm{php}$

[4] D. Folta, N. Bosanac, A. Cox, K.C. Howell, "The Lunar IceCube Mission Design: Construction of Feasible Transfer Trajectories with a Constrained Departure," AAS/AIAA Space Flight Mechanics Meeting, Napa Valley, CA, February 2016.

[5] D. Folta, N. Bosanac, A. Cox, K.C. Howell, "The Lunar IceCube Mission Challenge: Attaining Science Orbit Parameters from a Constrained Approach Trajectory," AAS/AIAA Space Flight Mechanics Meeting, San Antonio, TX, February 2017.

[6] Busek.com, "3cm RF Ion Thruster", Accessed January 2016: http://www.busek.com/

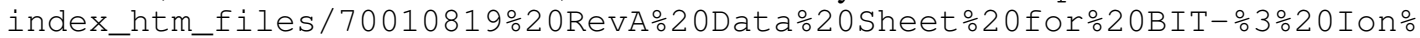
20 Thruster.pdf

[7] T. A. Pavlak, "Trajectory Design and Orbit Maintenance Strategies in Multi-Body Dynamical Regimes," Ph.D. Dissertation, School of Aeronautics \& Astronautics, Purdue University, West Lafayette, IN, May 2013.

[8] B.A. Conway, Spacecraft Trajectory Optimization, New York: Cambridge University Press, 2010.

[9] STK 10 Help, Accessed August 2015: http://www.agi.com/resources/help/online/ stk/10.1/index.html

[10] N. Bosanac, "Leveraging Natural Dynamical Structures to Explore Multi-Body Systems," Ph.D. Dissertation, School of Aeronautics \& Astronautics, Purdue University, West Lafayette, IN, August 2016.

[11] Lunar IceCube to Take on Big Mission From Small Package, Accessed January 2017: https://www.nasa.gov/feature/goddard/ lunar-icecube-to-take-on-big-mission-from-small-package

[12] W.M. Folkner, J.G. Williams, D.H. Boggs,"The Planetary and Lunar Ephemeris DE 421," IPN Progress Report, Vol. 42-178, 2009, pp. 1-34.

[13] SPICE Toolkit, Accessed May 2016: https://naif.jpl.nasa.gov/naif/aboutspice. html

[14] A. Haapala, "Trajectory Design in the Spatial Circular Restricted Three-Body Problem Exploiting Higher-Dimensional Poincaré Maps," Ph.D. Dissertation, School of Aeronautics \& Astronautics, Purdue University, West Lafayette, IN, December 2014. 